\title{
A Conversational Model to Display User Activity
}

\author{
Tina Deiml-Seibt \\ University of Applied \\ Sciences Potsdam \\ Interaction Design Lab \\ Pappelallee 8-9, 14469 \\ Potsdam, Germany \\ deiml@fh-potsdam.de
}

\author{
Larissa Pschetz \\ University of Applied \\ Sciences Potsdam \\ Interaction Design Lab \\ Pappelallee 8-9, 14469 \\ Potsdam, Germany \\ larissa.pschetz@fh- \\ potsdam.de
}

\author{
Boris Müller \\ University of Applied \\ Sciences Potsdam \\ Interaction Design Lab \\ Pappelallee 8-9, 14469 \\ Potsdam, Germany \\ boris.mueller@fh- \\ potsdam.de
}

\begin{abstract}
The creation of mechanisms to motivate user participation became a necessary strategy for evolution and sustainability of many online systems. Nowadays, most of these mechanisms are based on a form of displaying user activity, e.g. through badges or ranking scores. In this paper we discuss the current use of activity displays as incentives for user participation, and present IntroText, an alternative conversational approach. Based on the metaphor of introducing someone, IntroText sketches a behavioral portrait of users in an individual and non-competitive way. We describe the implementation of this feature pointing out to its potential to influence online behavior.
\end{abstract}

\section{Categories and Subject Descriptors}

H.5.3 [Information Interfaces and Presentation]: Group and Organization Interfaces

\section{General Terms}

Human Factors, Interface Design

\section{Keywords}

Social software, incentive, user activity, tracking

\section{INTRODUCTION}

Nowadays, online communities and the concept of "Architecture of Participation" [14] are widespread. Systems are designed to encourage and, at the same time, profit from users' participation. Online communities can be very helpful in this task: their members generate content, attract other users, and are likely to return to the website. The creation of a stable community, however, is not a simple task. In order to hold the community together, creating a website that allows users to register and contribute is not enough. Facing high competition, systems started to create various

(C) The Author 2009.

Published by the British Computer Society mechanisms in order to get users involved in their causes. Analyzing users' engagement, researchers realized that contributions can be considered as a way of drawing attention from the community $[8,3]$, or the data resulting from members' communication [14]. Thus, displaying users' activity became a common way of stimulating participation.

Displays of user activity can take a variety of forms. A clear difference can be noticed between systems whose communities are formed around a theme (news, recipes, books, etc.) and systems whose argument is the social network itself $^{1}$. While the second tends to directly display single activities and updates within a social group, the first tends to translate activities into a sort of ranking code, through which some users fill leadership roles and others do not.

Ranking users based on their activities is a good method to promote active users within a community, but if the aim is to stimulate participation in the community as a whole, it might be controversial. Nowadays, it is known that ranking activity in online communities follows a power law $[2,14]$. It has been shown, for example, that few contributors produce most of the content in wikipedia [15], and few blogs attract the great majority of readers [16]. Thus, it can be concluded that very few users will reach top levels of contributions. In this case, showing activity levels in a hierarchical way - expecting that most of the community will struggle to evolve in the established social hierarchy - can be a recipe for disappointment. Furthermore, even if all users take the challenge of evolving in the hierarchy, they are likely to give up after certain time.

In this paper we discuss ways of displaying user participation (Section 2), and present IntroText (Section 3), an activity display designed to enhance participation within a community of interest in the architectural domain. The interface feature builds on the social practices of status updating and aims to be an alternative for ranking models. Its implementation not only encourages further studies (Section 5) but also raises issues related to privacy and the non-intentional creation of user related metadata (Section 4).

\section{OVERVIEW AND RELATED WORK}

Psychology studies [11] have shown that the motivation to contribute to online communities can spring from a variety of sources: from the expectation of reciprocity, to a sense of efficacy, or the sustainment of one's reputation. Since all these factors are intrinsically related to the visibility of

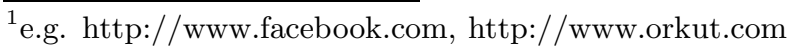


the contributed content within the community, putting contributions in evidence and tying them to their authors are essential for any incentive mechanism. Though the way of displaying user activity depends on the community, the architecture and the information presented by each system, some patterns and tendencies can be recognized.

\subsection{Ranking user activity}

In communities formed around a theme, ranking systems ${ }^{2}$ are the most common way of displaying user activity, and intend to: 1) reward active users, 2) establish activity paradigms and/or 3) help users track their own actions within the established model. Such mechanisms allow users to rate others' contributions (by giving stars, classifying as good or bad, etc), besides most of the times tracking the amount of contributions automatically (e.g. by calculating number of posts, comments, replies, etc.). The scores are translated into positions of a ranking scheme - automatically granting users a hierarchical profile. In this case, users are motivated to contribute in order to reach the next higher profile in the hierarchy, or to shape their behavior according to what they consider as a good paradigm.

This approach, however, presents an intrinsic contradiction. Nowadays, it is known that power law distributions tend to arise in any social system where many people express preferences among many options $[2,9]$. In such distributions the value of the Nth position is equivalent to $1 / \mathrm{N}$, which means the value of the second place corresponds to half of the first place, the value of the tenth place corresponds to one-tenth of the first place, etc. In this case, increasing the size of the system will consequently increase the gap between the first and the median place. Once the ranking of profiles or posts is consequently governed by power laws, very few users will be awarded high profiles, while the great majority will end up aiming at leadership roles without success. Such approach can therefore improve activity for a while and within some groups, but it is most likely to bring discontentment or to be ignored after a certain period.

Furthermore, if users take too long to evolve in a predefined hierarchy, they are likely to give up before making any evolution. One way of dealing with this problem, presented by Farzan et al.[5], is to establish different low and median roles so that little contribution would be enough for users to evolve to the next median role. This would make them realize their evolution and keep participating, even though they may never reach the highest profiles. On the other hand, if parameters to classify top roles are lowered, there is the risk of discouraging high contributors [13].

\subsection{Kinds of user activities}

Another common way of displaying user activity is to award badges or titles that represent the nature of users' actions $^{3}$. Such mechanism requires contributions to happen in different ways (e.g. tagging, commenting, etc). The clear definition of profiles, based on these actions, leads users to recognize each other within the community, motivating them to pursue and assume one of these profiles. In this approach the desired profile might be easily reached. However, if the

${ }^{2}$ Different ranking systems have been recognized as recurrent design solutions to stimulate user participation, and have been registered at the Yahoo Design Pattern Library (http://developer.yahoo.com/ypatterns/index.php)

${ }^{3}$ e.g. in http://stackoverflow.com/badges activities, and consequently the roles, are not varied enough, the badges might not be enough to differentiate users within the community and other ways of coping with their necessity of personalization might be necessary.

Incentive mechanisms are effective as long as they allow users to identify others and position themselves according to the created social systems. In this sense, the personalization of profiles is crucial. In the digital world, people cannot rely on social signs that we unconsciously follow in the physical world. So it is necessary to develop systems that provide cues about the presence and activity of users $[1,7,4]$.

\subsection{Activity status}

Another way of stimulating participation is to divulge activities of users within the social groups they belong. Facebook, for instance, openly reports the last activities of users to their friends through status messages. In this system, for example, a user $\mathrm{A}$ is informed that a friend $\mathrm{B}$ became friend of a user $\mathrm{C}$, which is consequently a potential friend of $\mathrm{A}$, or the same user A is informed that the friend $\mathrm{B}$ has tested a new Facebook application, which might therefore be an interesting suggestion for A. In this case the fact that friends have carried out a certain action is likely to inspire users to perform the same activity, thus encouraging participation.

Another tendency is to allow users to post mood messages. Here, users publish what they are doing, or how they feel at a specific moment to members of their social groups. These messages are a way to indirectly keep in contact with friends while personalizing one's profile. They are the basis of instant microblogging systems, such as Twitter ${ }^{4}$, and a characteristic of new ways of communication. By analyzing communities of Twitter users, for example, Java et al. [10] found out that the largest most common use of twitter is to talk about daily routine or current actions.

This approach, however, is hardly adaptable to non social network systems. First, it demands users to have a group of acquaintances - among which updates would be exchanged. Second, for the automatic update, the system must offer a variety of activities or each activity must allow enough variation to generate novel updates. Finally, the mood messages may disturb or may not be understood by those that are not close friends.

\section{INTROTEXT}

Based on the previous analysis we developed IntroText, an interface feature to improve users' participation in the MACE platform. The platform is an interface to access resources from different European repositories via metadata in the thematic area of Architecture. It not only allows retrieval and connection of contents from different repositories, but also supports a range of ways to contribute resources and metadata - which includes tagging, commenting, rating, adding content location, specifying field competence, as well as adding or linking content from external sources. Besides viewing, any action carried out over a resource consequently defines a link to this resource in the user page - so that it will stay available as a bookmark on the user page.

At the moment, the MACE community consists of about 150 registered users, one third of which is composed by technology researchers directly or indirectly working in the European project. The other two thirds consist of users related

\footnotetext{
${ }^{4} \mathrm{http}: / /$ twitter.com
} 


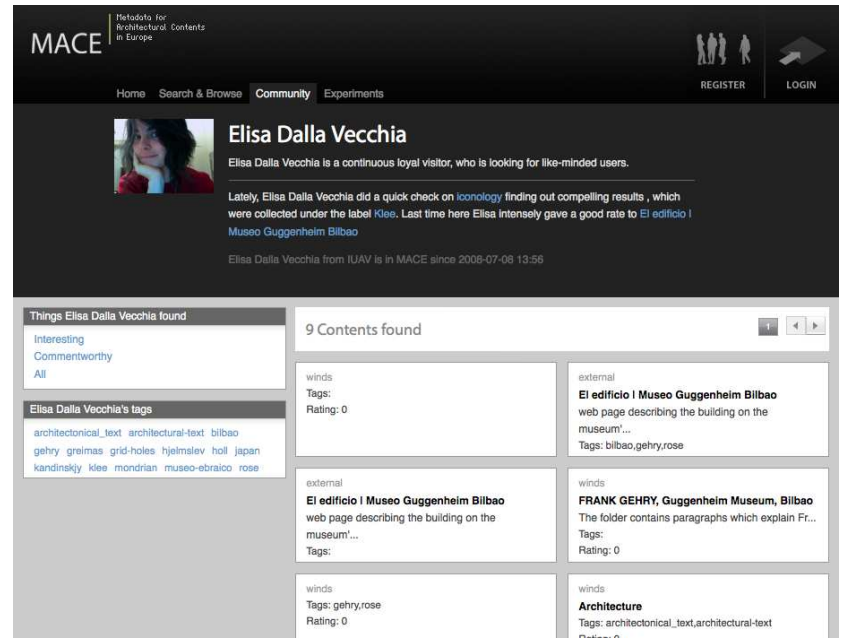

Figure 1: IntroText on profile page

to the architectural target group, whereby the majority are students. Their contribution is crucial, not only to maintain the system, but also to attract other users and repositories.

As part of a research project ${ }^{5}$, the platform is a place to try different strategies for metadata creation and community studies. For this reason, users can agree with the tracking of their actions in the system when they register. Tracking users' actions is a common practice in systems that have a sort of mechanisms to reward participation. The data gathered by tracking users' actions in MACE is the basis for IntroText.

Building on the metaphor of "introducing" somebody, IntroText aims at boosting activity through a conversational and non-hierarchical model. In the real world, when an individual enters the presence of others, they seek to acquire information about him/her or to bring into play information about him/her already established [6]. The ritual of introducing someone is a way of offering a starting point for this investigation.

In the online world the scenario is similar, by visiting a profile page users look for information that can facilitate the identification of this user, positioning themselves in relation to him/her. Having this in mind we designed IntroText to interpret user activity data and automatically generate statements that personalize users' profiles and portrays them within the system (see Figure 1).

IntroText evaluates activity within three different temporal degrees, and presents three kinds of sentences: a general description of the behavior of the user since registering, a description of recent behavior and a description of the last action in the system.

\subsection{Introducing Users}

In order to build IntroText, the following data is analyzed: day of last action (d), number of visits (n), number of days from registering until day of last action ( $\mathrm{t}$ ), number of tagged contents, number of rated contents, number of days passed since last action (dp), most frequent searched

${ }^{5} \mathrm{MACE}$ is a research project, founded by the European Union over the eContentplus programme, to interconnect and disseminate digital information about architecture over Europe repository, visit frequency $(\mathrm{n} / \mathrm{t})$, number of visits/number of tagged contents (n/tg) and number of visits/number of rated contents $(\mathrm{n} / \mathrm{rt})$. This data was used to define introductory sentences regarding three temporal levels: long-term, midterm and last visit behaviors.

In the long-term level, general behaviors are defined based on users' visit frequencies (active time/time since registered) and time passed since last actions. This data is interrelated and transformed in phrases such as: "eventually shows up", "is on vacation", "is a continuous loyal visitor", "had fun for a day", etc. If recurrent actions are recognized, they are added to the phrase as favorite occupations. For instance, if the main action is tagging, the system outputs the expression "who has made valuable contributions to the organization of contents". If adding new resources is the main action, it prints "who spends most of the time contributing new resources", and so on.

For the outline of mid-term and last visit behavior, IntroText analyzes action sequences such as entered search terms - number of results viewed - time spent throughout the search - action performed on a viewed content (tagging, commenting, viewing). These actions are translated into sentences that suggest the theme in which the user is currently involved, e.g. "Lately \{user name $\}$ thoroughly browsed classification terms", "Quite a while ago, \{user name $\}$ briefly labeled \{tagged resource\} with \{given tag\}", "Last time here, \{user name $\}$ quickly found \{checked resource $\}$ while searching for $\{$ searched term $\} "$ (see Figure 2 examples).

Each temporal level presents a different set of possible sentences. Though they are likely to be expanded in the future, at the moment, the long-term behavior level includes two group of sentences: one offering 25 and the other 15 phrases possibilities; the mid-term level has three groups of 5 possibilities and the last visit level has three groups of 2 , 4 and 6 possibilities. All together we have 67 sentences that allow for 2250000 different profiles descriptions. In fact, the variety of sentences and combinations is even higher, once the mid-term and last visit descriptions include tagged and searched terms, as well as viewed resources, which are likely to vary from one user to another even if the same sentence structure is presented. Special attention was dedicated to sentences describing low activity levels. Among the sentences defined, 34 describe very low levels of activity.

With the help of IntroText, activity is stimulated in order to communicate with others. By visiting one's profile it is possible to know the topics in which this user has been recently and/or constantly interested, and to realize how long he/she has been involved with such topics. The general description based on the long-term analysis gives a hint to the amount and kind of resources that can be found in the profile page of each user. Adverbs and adjectives, like quickly, intensely or cautious are used to form a perception of users' momentary moods. Most recently searched terms, ratings, comments, given tags or added resources inform about user's research topics, the way they perform research and their preferred actions and contents. IntroText also indirectly informs about possible ways to handle research with the help of MACE tools. It inspires curiosity without being frustrating, because it presents no goals or competition levels to be achieved.

Though MACE only includes academic and educational content, we understand that the divulgation of metadata produced by users in a non-intentional way (such as most 

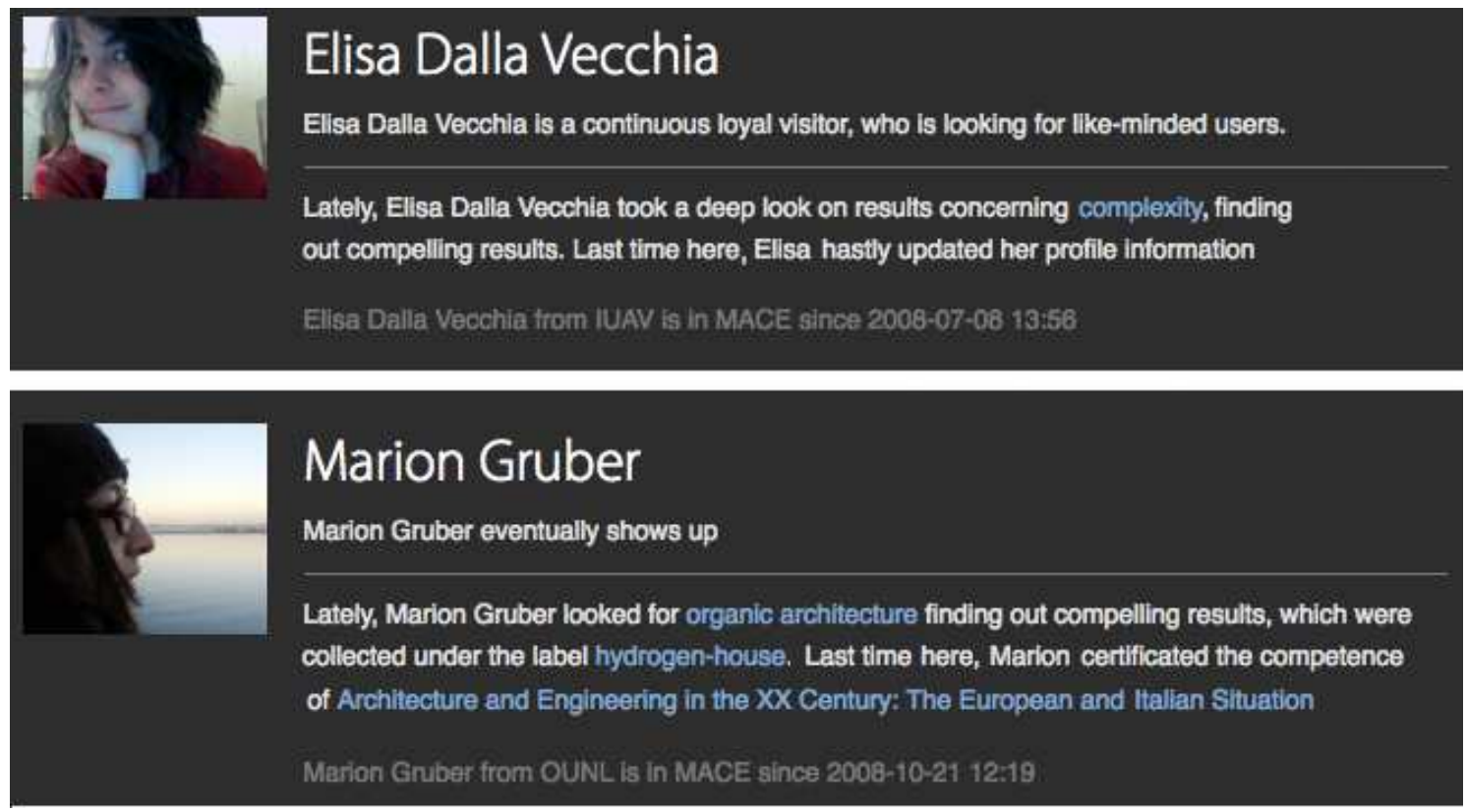

Figure 2: IntroText examples

performed actions and most entered searched terms) could change search behaviors or lead to a feeling of privacy violation. Allowing users to deactivate default features also doesn't help much, since it is known that most users don't change the default settings when registering to a system ${ }^{6}$. In order to better understand the effects of the divulgation of such data, we carried out a short questionnaire, as presented in the next section.

It is interesting to remark that the tension between privacy and visibility assumes a crucial role here. It is known that what people say and do with another person depends on who, and how many, are watching [4]. As students are asked by their professors to access the website, they are likely to focus searches on themes considered as valuable within the community. The divulgation of non-intentional metadata, however, is not an innovation, other systems are fully based on $\mathrm{it}^{7}$, and some even claim it to be able to define more reliable user profiles.

\section{INTROTEXT IMPLEMENTATION}

IntroText has been implemented on the 29th of January 2009, as the first step of a wider community experiment. During the first month of implementation some changes in the tracked data could already be perceived ${ }^{8}$ (see Figure 3 ). For instance, a slight increase in the visits of users profile was identified. We assume that, as IntroText is continuously changing, users return to the system not only to check profiles of other users, but also to verify updates in their own profile pages. Additionally, a slight increase in all activities

\footnotetext{
${ }^{6} \mathrm{~A}$ study about privacy in online networks, for example, found out that more than $99 \%$ of the Twitter users retained the default privacy settings upon their profiles [12]

${ }^{7}$ e.g. Cluztr: http://www.cluztr.com

${ }^{8}$ Activity of MACE users started to be tracked one month after the portal has been launched on September 2008, and about 3 months before the implementation of IntroText
}

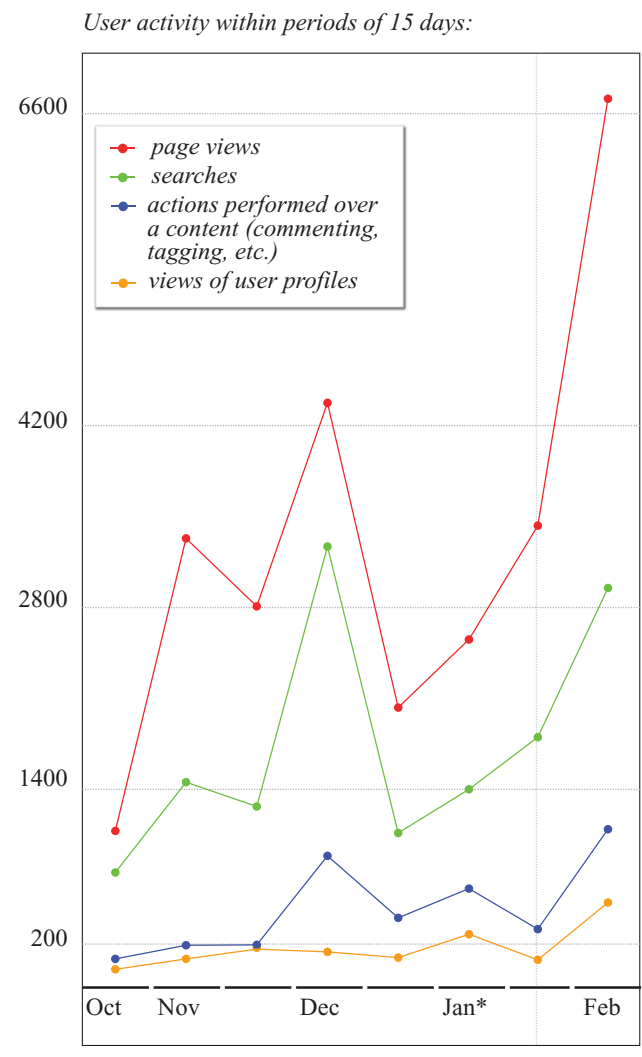

* the avarage of the second half of January was measured until the 29th (the day of the implementation of the IntroText)

Figure 3: Graph displaying evolution of users' activities in MACE before and after the implementation of IntroText 


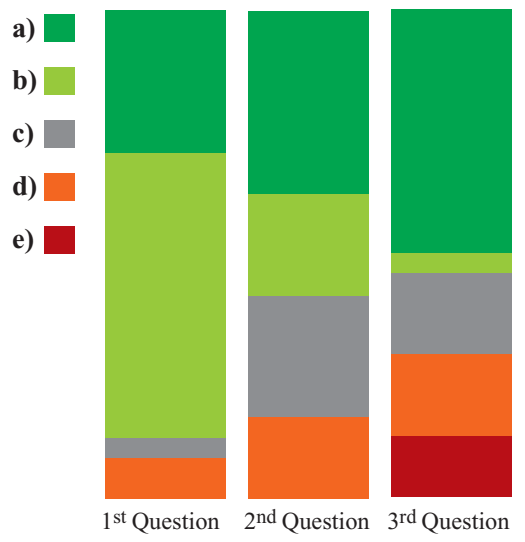

Figure 4: Results of the survey carried out with MACE users, green represents positive answers, red and orange negative ones

(regarding page views, actions and searches) could be observed, which might be caused by users trying out different ways of shaping their introductions.

Three weeks after the implementation of IntroText, we carried out a questionnaire composed by three multiple questions. In order to evaluate users' concerns on privacy issues involved in the divulgation of metadata produced in a nonintentional ways. The first question asked about their general impression of the new feature, offering the options: a) is great, I enjoyed it / b) is somehow funny / c) has nothing to do with me on MACE / d) bothers me a bit / e) is insulting. The second and third questions asked how they felt about the possibility of having others seeing their most performed actions (tagging, commenting, etc.) and their most entered searched terms on MACE, both questions presented the same alternatives for answering: a) I'm glad to share / b) I even enjoy it / c) I don't mind / d) It eventually bothers me / e) I don't like it / f) Other... (please tell me). The questionnaire was sent to all registered users, 24 answers returned and are illustrated on the graph of Figure 4.

Results from this preliminary survey were mostly positive. $87,5 \%$ of the answers evaluated the feature as great or amusing, only $16,6 \%$ did not support the communication of most performed actions (tagging, commenting, etc.), and 29,2\% disapproved the display of entered search terms.

\section{FUTURE WORK}

In the near future IntroText will be monitored and refined iteratively. In order to improve results, however, additional community features must be improved or implemented in the MACE portal, such as ways of contacting users and an enhanced access to users' profile pages. At the time IntroText was launched, the viability and suitability of many features were under study, being not yet implemented - such as an adaptive interface through which logged users would see other matching profiles, or the possibility of automatically receiving updates of previously defined users' activities.

\section{ACKNOWLEDGMENTS}

This project was co-funded under contract number ECP 2005 EDU 038098 in the eContentplus programme in the context of the MACE project.
We would like to thank all the Mace users for their feedback and participation in the survey, and our colleagues Till Nagel, Moritz Stefaner and Martin Memmel for their construtive feedback.

\section{REFERENCES}

[1] M. S. Ackerman and B. Starr. Social activity indicators: interface components for cscw systems. In UIST '95: Proceedings of the 8th annual ACM symposium on User interface and software technology, pages 159-168, New York, NY, USA, 1995. ACM.

[2] A.-L. Barabasi. Linked: How Everything Is Connected to Everything Else and What It Means for Business, Science, and Everyday Life. Plume Publishers, 2003.

[3] S. L. Bryant, A. Forte, and A. Bruckman. Becoming wikipedian: transformation of participation in a collaborative online encyclopedia. In GROUP '05: Proceedings of the 2005 international ACM SIGGROUP conference on Supporting group work, pages 1-10, New York, NY, USA, 2005. ACM.

[4] T. Erickson, D. N. Smith, W. A. Kellogg, M. Laff, J. T. Richards, and E. Bradner. Socially translucent systems: social proxies, persistent conversation, and the design of "babble". In CHI '99: Proceedings of the SIGCHI conference on Human factors in computing systems, pages 72-79, New York, NY, USA, 1999. ACM.

[5] R. Farzan, J. M. DiMicco, D. R. Millen, C. Dugan, W. Geyer, and E. A. Brownholtz. Results from deploying a participation incentive mechanism within the enterprise. In CHI '08: Proceeding of the twenty-sixth annual SIGCHI conference on Human factors in computing systems, pages 563-572, New York, NY, USA, 2008. ACM.

[6] E. Goffman. Presentation of Self in Everyday Life. Doubleday Anchor Books, New York, NY, USA, 1959.

[7] W. Hill, L. Stead, M. Rosenstein, and G. Furnas. Recommending and evaluating choices in a virtual community of use. In CHI '95: Proceedings of the SIGCHI conference on Human factors in computing systems, pages 194-201, New York, NY, USA, 1995. ACM Press/Addison-Wesley Publishing Co.

[8] B. Hoisl, W. Aigner, and S. Miksch. Online Communities and Social Computing, volume Volume 4564/2007 of Lecture Notes in Computer Science, chapter Social Rewarding in Wiki Systems Motivating the Community, pages $362-371$. Springer, Berlin / Heidelberg, 2007.

[9] B. A. Huberman. The Laws of the Web: Patterns in the Ecology of Information. The Mit Press, Cambridge, MA, USA, 2001.

[10] A. Java, X. Song, T. Finin, and B. Tseng. Why we twitter: understanding microblogging usage and communities. In WebKDD/SNA-KDD '0\%: Proceedings of the 9th WebKDD and 1st SNA-KDD 2007 workshop on Web mining and social network analysis, pages 56-65, New York, NY, USA, 2007. ACM.

[11] P. Kollock. Communities in Cyberspace, chapter The economies of online cooperation: Gifts and public goods in cyberspace., pages 220-239. Routledge, New York, NY, USA, 1999. 


\section{T. Deiml-Seibt et al.}

[12] B. Krishnamurthy and C. E. Wills. Characterizing privacy in online social networks. In WOSP '08: Proceedings of the first workshop on Online social networks, pages 37-42, New York, NY, USA, 2008. ACM.

[13] O. Nov. What motivates wikipedians? Communications of the ACM, 50(11):60-64, 2007.

[14] T. O'Reilly. What is web 2.0: Design patterns and business models for the next generation of software.
[15] F. Ortega, J. M. Gonzalez-Barahona, and G. Robles. On the inequality of contributions to wikipedia. In HICSS '08: Proceedings of the Proceedings of the 41st Annual Hawaii International Conference on System Sciences, page 304, Washington, DC, USA, 2008. IEEE Computer Society.

[16] C. Shirky. Power laws, weblogs and inequality. 